\title{
MiR-506 suppresses proliferation of hepatoma cells through targeting YAP mRNA 3'UTR
}

\author{
Yue WANG ${ }^{1}$, Ming CUI ${ }^{2}$, Bao-di SUN² ${ }^{2}$ Fa-bao LIU $^{1}$, Xiao-dong ZHANG ${ }^{2}$, Li-hong YE ${ }^{1, *}$ \\ ${ }^{1}$ State Key Laboratory of Medicinal Chemical Biology, Department of Biochemistry, College of Life Sciences, Nankai University, Tianjin \\ 300071, China; ' State Key Laboratory of Medicinal Chemical Biology, Department of Cancer Research, College of Life Sciences, \\ Nankai University, Tianjin 300071, China
}

\begin{abstract}
Aim: MiR-506 is a miRNA involved in carcinogenesis of several kinds of cancer. In this study, we explored whether miR-506 played a critical role in hepatocellular carcinoma (HCC).

Methods: Twenty HCC and adjacent normal liver tissue samples were collected. Human hepatoma cell lines HepG2 and H7402 were used for in vitro studies. The expression of miR-506 and transcriptional co-activator YAP was examined using qRT-PCR. Western blot analysis was used to measure the expression of YAP and its target genes. Luciferase reporter gene assay was used to identify YAP as a target gene of miR-506. MTT and EdU assays were carried out for functional analysis.

Results: The expression of miR-506 was significantly lower in HCC than in adjacent normal liver tissues. Bioinformatics analysis revealed that YAP mRNA might be one of the targets of miR-506, and miR-506 in HCC tissues was found to be negatively correlated with YAP ( $r=-0.605)$. In both HepG2 and H7402 cells, miR-506 dose-dependently down-regulated YAP and its target genes c-Myc and the connective tissue growth factor (CTGF). Luciferase reporter gene assays demonstrated that miR-506 targeted the wild type 3 'UTR of YAP mRNA, but not a 3'UTR with a mutant seed site. Furthermore, miR-506 significantly inhibited the proliferation of HepG2 and H7402 cells, while anti-miR-506 enhanced the cell proliferation, which was blocked by YAP siRNA.

Conclusion: MiR-506 suppresses the proliferation of hepatoma cells by targeting YAP mRNA.
\end{abstract}

Keywords: microRNA; miR-506; hepatocellular carcinoma; cell proliferation; YAP; c-Myc; connective tissue growth factor

Acta Pharmacologica Sinica (2014) 35: 1207-1214; doi: 10.1038/aps.2014.59; published online 4 Aug 2014

\section{Introduction}

MicroRNAs (miRNAs) are small, non-coding RNAs of approximately 19-25 nucleotides. MiRNAs negatively modulate gene expression through RNA-induced silencing complex-mediated mRNA cleavage or translational repression when partially complementary sequences are present in the 3' un-translated regions (3'UTRs) of target mRNAs ${ }^{[1,2]}$. Most importantly, numerous miRNAs are involved in cancers, and they can take on opposing roles as oncogenes or tumor suppressor genes depending on the target genes ${ }^{[3-5]}$. Hepatocellular carcinoma (HCC) is the sixth most common neoplasm and the third leading cause of cancer death worldwide $\mathrm{e}^{[6]}$. The development and progression of HCC is associated with the accumulation of additional genetic alterations ${ }^{[7]}$. Many studies have emphasized the causal links between miRNA deregulation and cancer development. Our laboratory has reported that miR-

\footnotetext{
* To whom correspondence should be addressed. E-mail yelihong@nankai.edu.cn

Received 2014-03-24 Accepted 2014-05-18
}

520e (or miR-205) suppresses the growth of hepatoma cells through the targeting of NF- $\mathrm{B}$-inducing kinase (NIK) mRNA (or hepatitis $B$ virus $X$ protein $\mathrm{mRNA})^{[8,9]}$. Recently, it has been reported that miR-506 inhibits epithelial mesenchymal transition (EMT) in ovarian cancer and breast cancer ${ }^{[10,11]}$. In addition, miR-506 can significantly restrain the proliferation of lung cancer cells ${ }^{[12]}$. All these reports suggest that miR-506 is a novel microRNA that is important in the development of cancer. However, the functions of miR-506 in liver cancer have not been well documented.

The Hippo pathway is closely associated with hepatocarcinogenesis $^{[13-15]}$. The most critical effector of the Hippo pathway is the transcriptional co-activator YAP. By co-activating key transcriptional factors, YAP plays a considerable role in regulating Hippo pathway-responsive gene ${ }^{[16,17]}$. Deregulation of this pathway leads to massive overgrowth of tissue and induces cancer ${ }^{[18]}$. More importantly, liver-specific overexpression of YAP results in a greater than 5-fold size enlargement that is reversible after cessation of YAP expression ${ }^{[16,19]}$. Previously, our group found that the expression levels of YAP were 
dramatically elevated in clinical HCC tissues, and that YAP was a key driver gene in $\mathrm{HBx}$-induced hepatocarcinogenesis in a CREB-dependent manner ${ }^{[20]}$. However, understanding of the post-transcriptional control of the YAP gene in liver cancer remains elusive.

In this study, we investigated the role of miR-506 in hepatocarcinogenesis. Interestingly, our findings show that miR-506 is able to suppress the proliferation of hepatoma cells through direct targeting of YAP mRNA. Our findings provide new insights into the mechanism by which miR-506 modulates cell proliferation in HCC.

\section{Materials and methods Patient samples}

Twenty paired HCC and adjacent non-tumorous liver tissues were collected from patients undergoing resection of HCC in Tianjin First Center Hospital (Tianjin, China). The relevant characteristics of the studied subjects are shown in Supplementary Table 1. Written consents approving the use of their tissues for research purposes were obtained from the patients. The study protocol was approved by the Institute Research Ethics Committee at the Nankai University.

\section{Cell lines and cell culture}

Human hepatoma cell lines, HepG2 and H7402 ${ }^{[20]}$, were maintained in Dulbecco's modified Eagle's medium and RPMI medium 1640, respectively (Gibco, CA, USA). Media were supplemented with heat inactivated $10 \%$ fetal bovine serum (FBS, Gibco, CA, USA), $100 \mathrm{U} / \mathrm{mL}$ penicillin and $100 \mathrm{mg} / \mathrm{mL}$ streptomycin in $5 \% \mathrm{CO}_{2}$ at $37^{\circ} \mathrm{C}$.

\section{Plasmid construction}

A 294 bp fragment of the YAP 3'UTR was sub-cloned into a pGL3-Control vector (Promega, Madison, WI, USA) immediately downstream of the stop codon of the luciferase gene to generate pGL3-YAP. A mutant construct of the YAP 3'UTR (named pGL3-YAP-MUT), carrying a substitution of 8 nucleotides within the core seed sequence of miR-506, was constructed using overlapping extension PCR. The primers used in this study for plasmid construction were as follows: pGL3-YAP forward, 5'-TGCTCTAGAGGGTATTCTTGAATTGCTTT-3', reverse, 5'-GGGGGCCGGCCGTCTTTCTGCCATGTTCCA-3'; and pGL3-YAP-MUT forward, 5' -TTTTCTAAATGTACACGGAAAAAAGGAAAAATGAACACA-3', reverse, 5'-TGTGTTCATTTTTCCTTTTTTCCGTGTACATTTAGAAAA-3'.

\section{Cell transfection}

The cells were cultured in a 6-well or 24-well plate for $24 \mathrm{~h}$ and were then transfected with plasmids or miRNAs. All transfections were performed using Lipofectamine 2000 reagent (Invitrogen, Carlsbad, CA, USA) according to the manufacturer's protocol. YAP siRNA, miR-506 (anti-miR-506), and miRNA control (miRNA Ctrl or anti-miRNA Ctrl) were synthesized by RiboBio (Guangzhou, China). The siRNA duplex sequences used were as follows: YAP siRNA, 5'-GACAUCUUCUGGUCAGAGAdTdT-3' ${ }^{[20]}$.

\section{Quantitative real-time polymerase chain reaction (qRT-PCR)}

Total RNA was extracted from the cells or tissues using TRIzol (Invitrogen, Carlsbad, CA, USA) according to the manufacturer's protocol. For mature miR-506 detection, total RNA was polyadenylated by poly(A) polymerase (Ambion, Austin, TX, USA) as described previously ${ }^{[8]}$. Reverse transcription was performed using poly(A)-tailed total RNA and reverse transcription primer with ImPro-II Reverse Transcriptase (Promega, Madison, WI, USA) according to the manufacturer's instructions. The qRT-PCR was performed as described in the method of Fast Start Universal SYBR Green Master (Rox) (Roche Diagnostics GmbH Mannheim, Germany). The primers used were as follows: YAP forward, 5' -TATCAATCCCAGCACAG-3', reverse, 5'-GGAATGGCTTCAAGGTAG-3'; GAPDH forward, 5'-CATCACCATCTTCCAGGAGCG-3', reverse, 5' -TGACCTTGCCCACAGCCTTG-3'; miR-506 forward, 5'-TAAGGCACCCTTCTGAGTAGA-3', reverse, 5'-GCGAGCACAGAATTAATACGAC-3'; and U6 forward, 5'-AGAGCCTGTGGTGTCCG-3', reverse, $5^{\prime}$-CATCTTCAAAGCACTTCCCT-3'.

\section{Luciferase reporter gene assays}

Luciferase reporter gene assays were performed using the Dual-Luciferase Reporter Assay System (Promega, Madison, WI, USA) according to the manufacturer's instructions. Cells were transferred into 24 -well plates at $3 \times 10^{4}$ cells per well. After $24 \mathrm{~h}$, the cells were transiently co-transfected with the pRL-TK plasmid (Promega, Madison, WI, USA) containing the Renilla luciferase gene, which was used for internal normalization, and with various constructs containing the seed sequence or mutant seed sequence of YAP 3'UTR, or pGL3-Control.

\section{Western blot analysis}

The Western blot analysis protocol has been described previously ${ }^{[20]}$. The primary antibodies used were mouse anti- $\beta$ actin (Sigma, St Louis, MO, USA), rabbit anti-YAP (Proteintech Group, USA), rabbit anti-CTGF (Proteintech Group, USA), and mouse anti-c-Myc (Santa Cruz Biotechnology, Santa Cruz, CA, USA). Protein bands were quantified using Quantity One software (Bio-Rad).

\section{Analysis of cell proliferation}

HepG2 cells were seeded onto 96-well plates (1000 cells/well) for $24 \mathrm{~h}$ before transfection, and the 3-(4,5-dimethylthiazol2-yl)-2,5-diphenyltetrazolium bromide (MTT) assay was used to assess cell proliferation every day from the first day until the third day after transfection. The protocol has been described previously ${ }^{[21]}$. The 5-ethynyl-2'-deoxyuridine (EdU) incorporation assay was carried out using the Cell-Light ${ }^{\mathrm{TM}}$ EdU imaging detecting kit according to the manufacturer's instructions (RiboBio). 


\section{Statistical analysis}

Statistical significance was assessed by comparing mean values (6 standard deviation; SD) using Student's $t$ test for independent groups and was assumed at $P<0.05$ and $P<0.01$ or reported as not significant (NS). The expression levels of miR506 in tumor tissues and matched adjacent non-tumor tissues were compared using Wilcoxon's signed-rank test. Pearson's correlation coefficient was used to determine the correlation between the expression levels of each gene in tumor tissues. Each experiment was repeated at least three times.

\section{Results}

MiR-506 is down-regulated and negatively correlated with YAP in HCC tissues

Deregulation of miRNA expression has been linked to tumor development and progression. It has been reported that miR506 shows a significant association with distant-relapse-free survival (DRFS) in more than $98 \%$ of breast cancer patients ${ }^{[11]}$. However, the expression of miR-506 in HCC remains unclear. First, we analyzed the expression of miR-506 in 20 paired clinical HCCs and adjacent non-tumor liver tissues using qRTPCR with normalization against an endogenous control (U6 RNA). Compared with matched non-tumor tissues, all 20 HCC samples showed significant down-regulation of miR-506 (Figure 1A), suggesting that reduced miR-506 expression is a frequent event in HCC tissues and may be involved in hepatocarcinogenesis. To explore the role of miR-506 in the development of liver cancer, we screened the target genes of miR-506 using Targetscan and microRNA.org (http:/ / www.targetscan. org/ and http://www.microrna.org). YAP was identified as a candidate, as it is an important onco-protein in liver cancer. Next, we examined the correlation between the expression of miR-506 and YAP in clinical HCC tissues. MiR-506 levels were markedly inversely correlated with YAP expression (Figure 1B), suggesting miR-506 might target YAP mRNA in HCC tissues. Therefore, we conclude that miR-506 is negatively correlated with YAP and is down-regulated in HCC tissues.

\section{MiR-506 is able to down-regulate YAP in hepatoma cells}

Next, we investigated the effect of miR-506 on YAP in hepatoma cells. HepG2 and H7402 cells were transiently transfected with miR-506. Interestingly, we observed that miR506 was capable of down-regulating YAP in the cells at the protein level in a dose-dependent manner (Figure 2A). The opposite result was obtained when the cells were treated with anti-miR-506 (Figure 2B), suggesting that miR-506 is able to suppress the expression of YAP in hepatoma cells. The transfection efficiency of miR-506 and anti-miR-506 was validated by qRT-PCR (Figure 2A and 2B). Furthermore, our data demonstrated that miR-506 down-regulated the expression levels of c-Myc and connective tissue growth factor (CTGF), two downstream target genes of YAP, in HepG2 and H7402 cells (Figure 2C) ${ }^{[22,23]}$. Conversely, anti-miR-506 heightened the expression levels of c-Myc and CTGF in the cells (Figure 2D). Therefore, we conclude that miR-506 is able to down-regulate YAP in hepatoma cells.
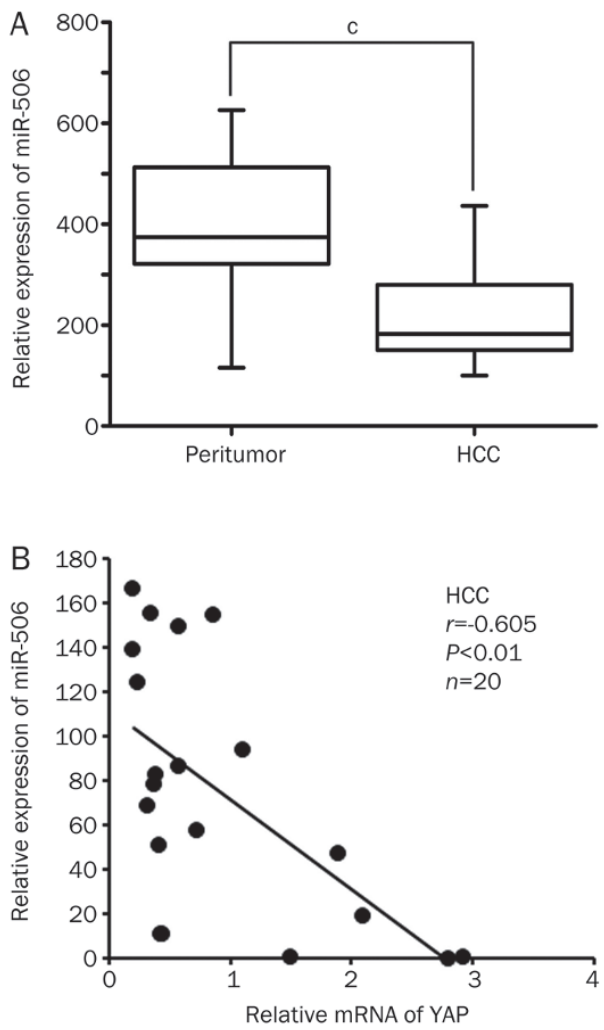

Figure 1. MiR-506 is down-regulated and negatively correlated with YAP in HCC tissues. (A) Relative expression levels of miR-506 were assessed by qRT-PCR in 20 pairs of clinical HCC tissues and corresponding nontumorous tissues ( ${ }^{c} P<0.01$, Wilcoxon's signed-rank test). (B) Correlation of miR-506 levels with YAP mRNA levels was examined by qRT-PCR in 20 cases of clinical HCC tissues ( $r=-0.605, P<0.01$, Pearson's correlation).

\section{MiR-506 restrains the expression of YAP through direct targeting} of YAP MRNA 3'UTR

To gain insight into the mechanism by which miR-506 inhibits YAP, we identified the miR-506 binding site in the YAP mRNA 3'UTR (Figure 3A) and constructed pGL3-YAP and pGL3YAP-MUT plasmids (Figure 3B). Our data showed that the co-transfection of miR-506 significantly suppressed the firefly luciferase activity of pGL3-YAP but failed to influence the luciferase activity of pGL3-YAP-MUT in HepG2 and H7402 cells (Figure 3C). Furthermore, inhibition of endogenous miR506 in the cells with anti-miR-506 resulted in increased firefly luciferase activity of the wild-type reporter but the mutant reporter (Figure 3D). Thus, our data indicate that miR-506 can attenuate the expression of YAP through direct targeting of its mRNA 3'UTR in hepatoma cells.

\section{MiR-506 suppresses the proliferation of hepatoma cells by} inhibiting YAP

To examine the potential role of miR-506 in tumorigenesis, we sought to determine whether miR-506 affects the proliferation of hepatoma cells using MTT and EdU assays. Our data indicated that the proliferation of HepG2 and H7402 cells was decreased when the cells were treated with miR-506, while the 
A

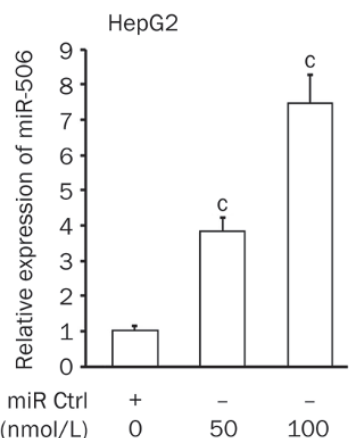

B

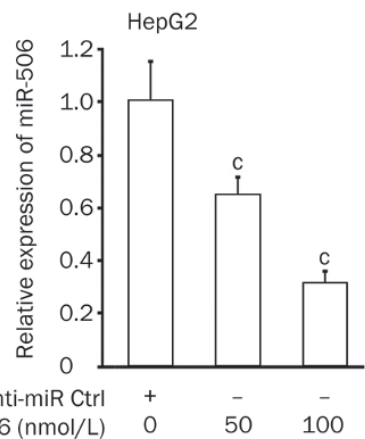

C

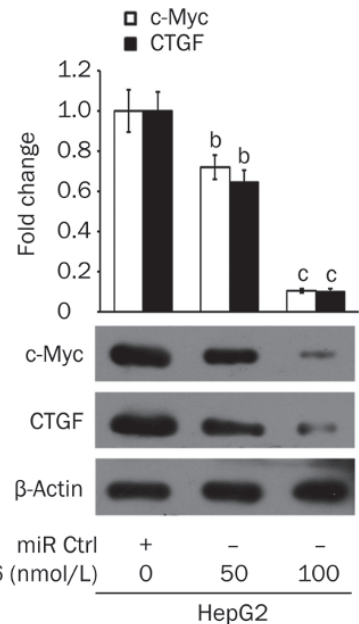

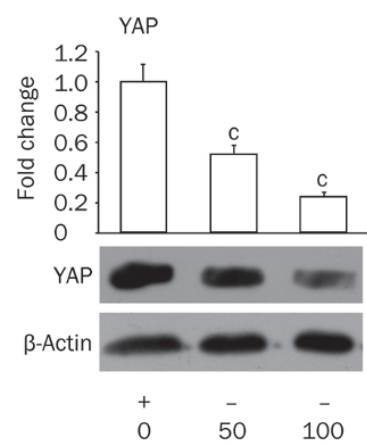

YAP
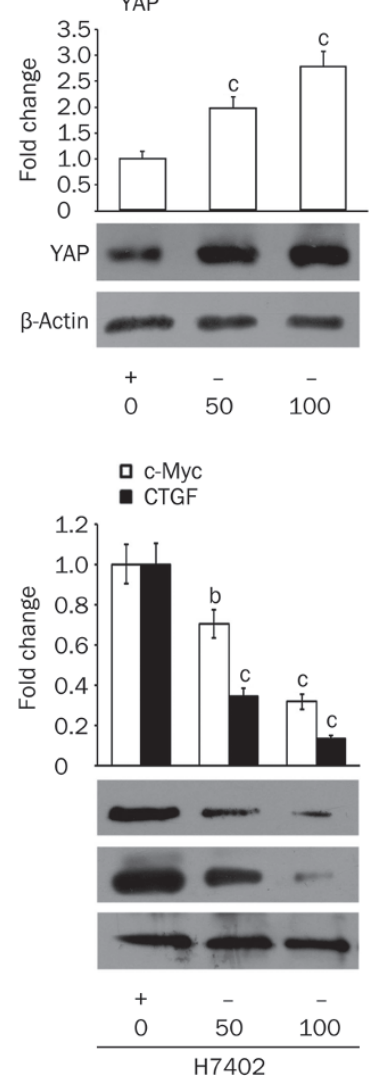

$\mathrm{H} 7402$

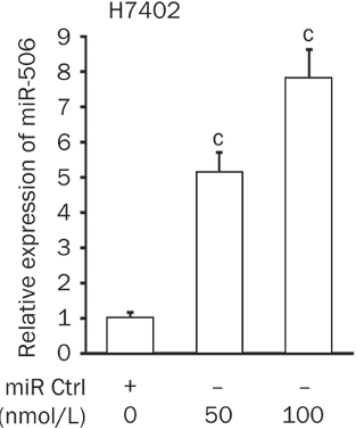

$\mathrm{H} 7402$

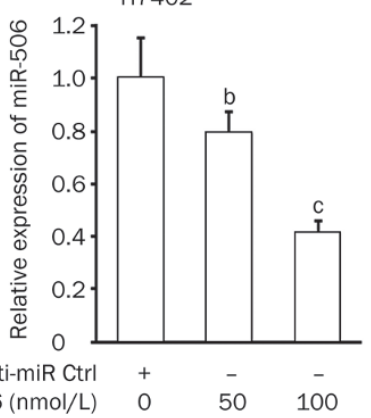

D

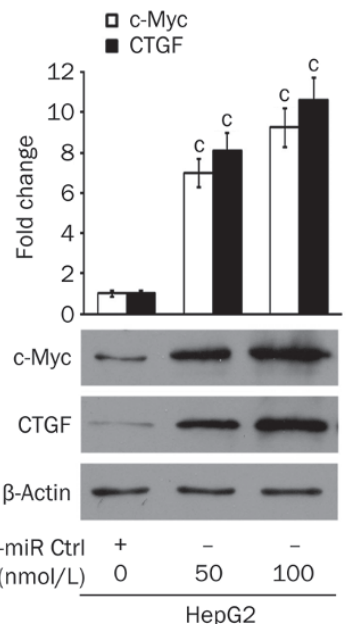

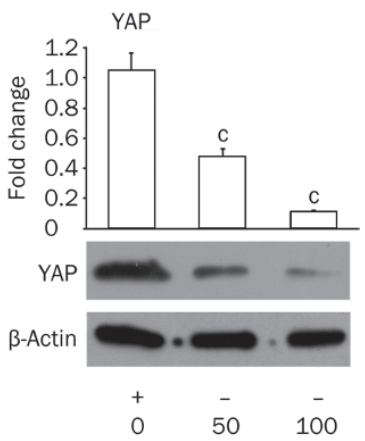
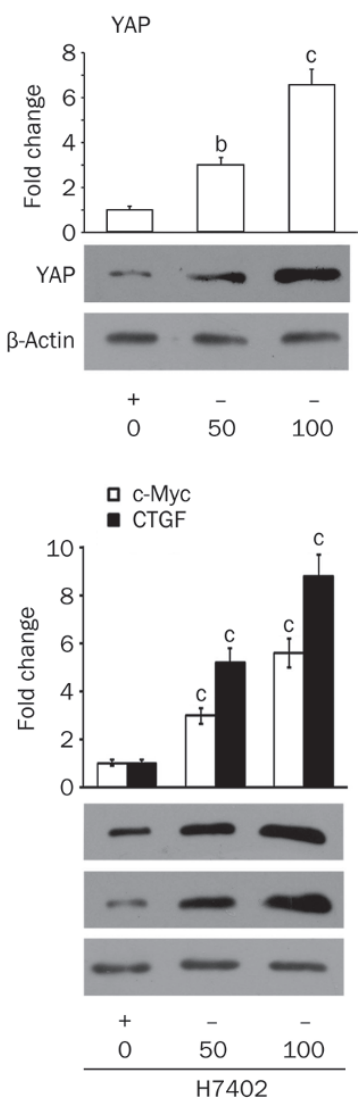

Figure 2. MiR-506 is able to down-regulate YAP in hepatoma cells. (A) The effect of miR-506 on YAP was examined by Western blot analysis in HepG2 and $\mathrm{H} 7402$ cells. Levels of miR-506 in the cells were validated by qRT-PCR. (B) The effect of anti-miR-506 on YAP was examined by Western blot analysis in HepG2 and H7402 cells. Levels of miR-506 in the cells were validated by qRT-PCR. (C) The effect of miR-506 on c-Myc and CTGF was examined by Western blot analysis in HepG2 and H7402 cells. (D) The effect of anti-miR-506 on c-Myc and CTGF was examined by Western blot analysis in HepG2 and $\mathrm{H} 7402$ cells. Statistically significant differences are indicated: ${ }^{\mathrm{b}} P<0.05 ;{ }^{\mathrm{c}} P<0.01$; Student's $t$ test.

treatment with anti-miR-506 increased the proliferation. The treatment with YAP siRNA was able to block the anti-miR506-enhanced proliferation (Figure 4), suggesting that miR-506 suppresses the proliferation of hepatoma cells by modulating YAP. Therefore, our observations suggest that miR-506 is able to inhibit the proliferation of hepatoma cells through direct targeting of YAP mRNA.

\section{Discussion}

As a heterogeneous tumor, HCC develops through the activation of multiple pathways and molecular alterations ${ }^{[24,25]}$. The development and progression of HCC is a complicated process that involves the deregulation of multiple genes that are essential to cellular biological processes. In this study, we are interested in the role of miR-506 in hepatocarcinogenesis.

We first investigated the expression levels of miR-506 in 20-paired clinical HCCs and adjacent non-tumor liver tissues. Interestingly, we observed that the expression levels of miR-506 were remarkably decreased in all 20 HCC samples relative to paired non-tumor tissues. Base on this finding, we hypothesized that miR-506 might be a novel tumor-suppressor miRNA in liver cancer. We screened the target genes 
A

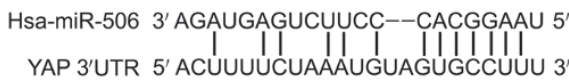

C
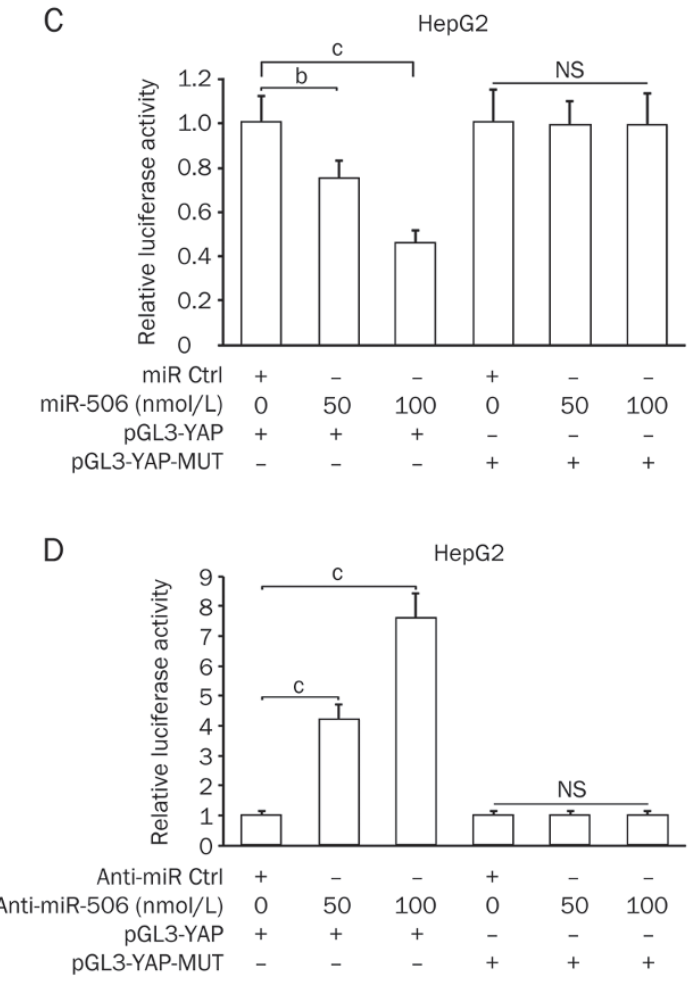

B

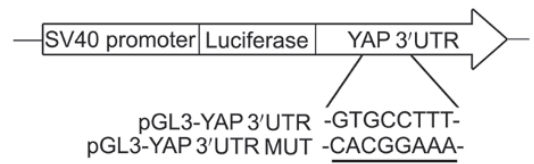

H7402

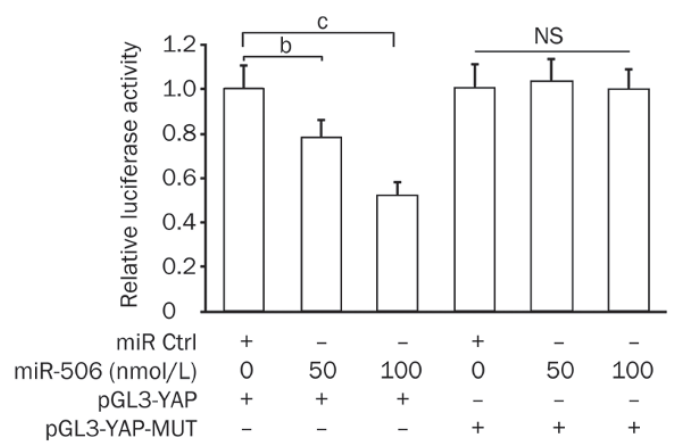

H7402

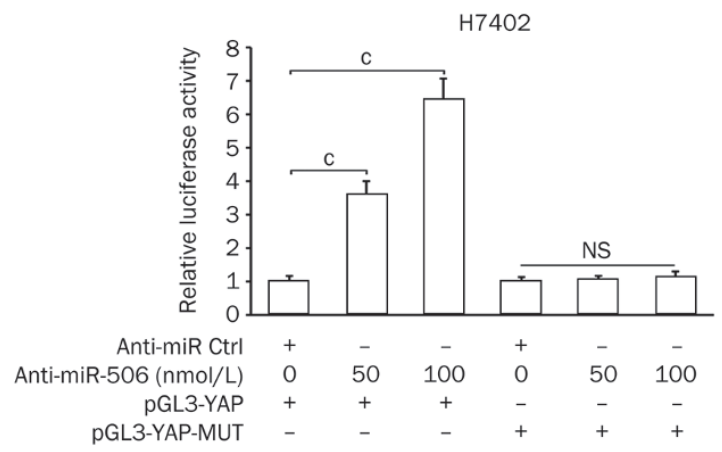

Figure 3. MiR-506 restrains the expression of YAP through direct targeting of YAP mRNA 3'UTR. (A and B) Model shows the binding site of miR-506 in the YAP mRNA $3^{\prime} U T R$ by bioinformatics prediction. Schematic diagram shows the generated mutant site in the YAP $3^{\prime} U T R$ seed region binding to miR-506 and the insertion sites of wild type YAP 3'UTR (or mutant) downstream of the luciferase reporter gene in pGL3-Control vector. (C) The effect of miR-506 on pGL3-YAP and pGL3-YAP-MUT reporter plasmids in HepG2 and H7402 cells was measured by luciferase reporter gene assays. (D) The effect of anti-miR-506 on pGL3-YAP and pGL3-YAP-MUT reporter plasmids in HepG2 and H7402 cells was measured by luciferase reporter gene assays. Statistically significant differences are indicated: ${ }^{\mathrm{b}} P<0.05,{ }^{\mathrm{C}} \mathrm{P}<0.01$; Student's $t$ test.

of miR-506 using bioinformatics tools. Form this screening, YAP stood out based on its particular functions and expression patterns. However, it had not been reported whether miR-506 could directly target YAP mRNA in HCC. Based on the bioinformatics analysis, we examined the correlation between the expression levels of miR-506 and YAP in clinical HCC tissues. As expected, we observed that miR-506 levels were inversely correlated with YAP expression in the tissues. We then further investigated the effects of miR-506 on YAP expression in hepatoma cells. Our data demonstrated that miR-506 was able to down-regulate YAP and its direct target genes (c-Myc and CTGF) in hepatoma cells. Moreover, we identified that miR-506 could directly bind to the $3^{\prime}$ UTR of YAP. We demonstrated that miR-506 could suppress the proliferation of hepatoma cells through direct targeting of YAP mRNA in functional assays.

YAP, an essential downstream effector of the Hippo pathway, is frequently highly expressed in a wide spectrum of human solid tumors ${ }^{[26,27]}$. It has been reported that YAP promotes proliferation in liver by regulating the transcrip- tion of certain target genes, including Ki-67, CTGF, c-Myc, sex-determining region Y-related high-mobility group box 4 (SOX4), H19, and alpha-fetoprotein (AFP) ${ }^{[1,20,28]}$. In clinical studies, YAP is an independent predictor of poor disease free survival (DFS) and overall survival (OS) in $\mathrm{HCC}^{[29]}$. Identifying the underlying mechanisms of YAP regulation will help facilitate the study of YAP as a novel target for the treatment of cancer. It has been reported that mitogen-activated protein kinase kinase 1 (MEK1) promotes YAP expression and that their interaction is critical for tumorigenesis in liver cancer ${ }^{[30]}$. In addition, there is a novel positive auto-regulatory feedback loop that underlies the interaction between YAP and c-Myc in liver cancer, which suggests that YAP and c-Myc link the Hippo-YAP and c-Myc pathways ${ }^{[13]}$. Therefore, as an oncogene, YAP plays crucial roles in the development of HCC. In this study, we enriched the understanding of the regulation mechanism of YAP at the post-transcriptional level.

Growing evidence suggests that alteration of microRNAs contributes to the development of cancer ${ }^{[31-33]}$. Recently, it has been reported that ABCC4, which is involved in the regula- 
A

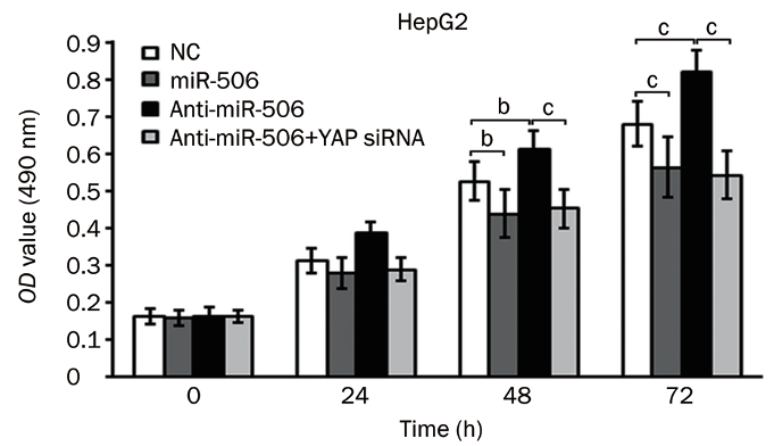

\section{B}
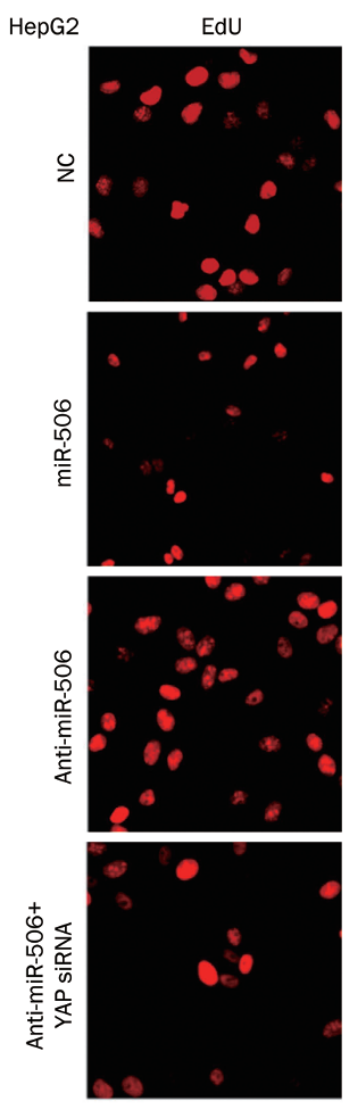

Hoechst
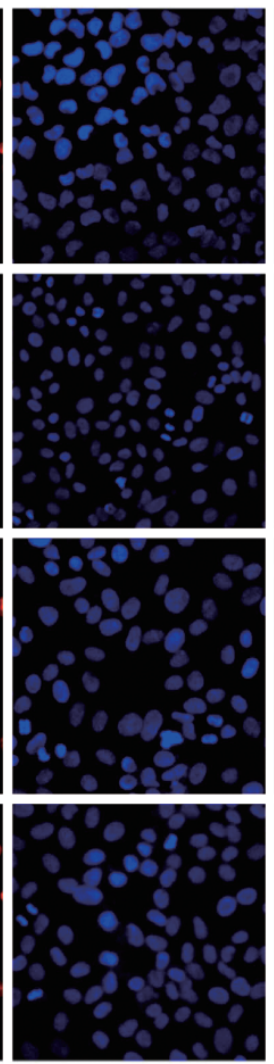

Merge
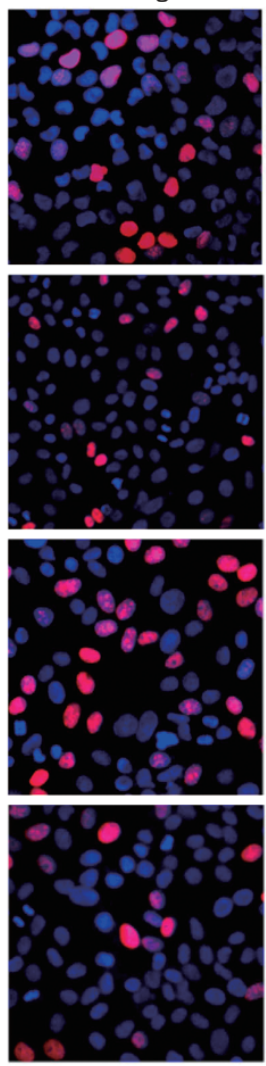
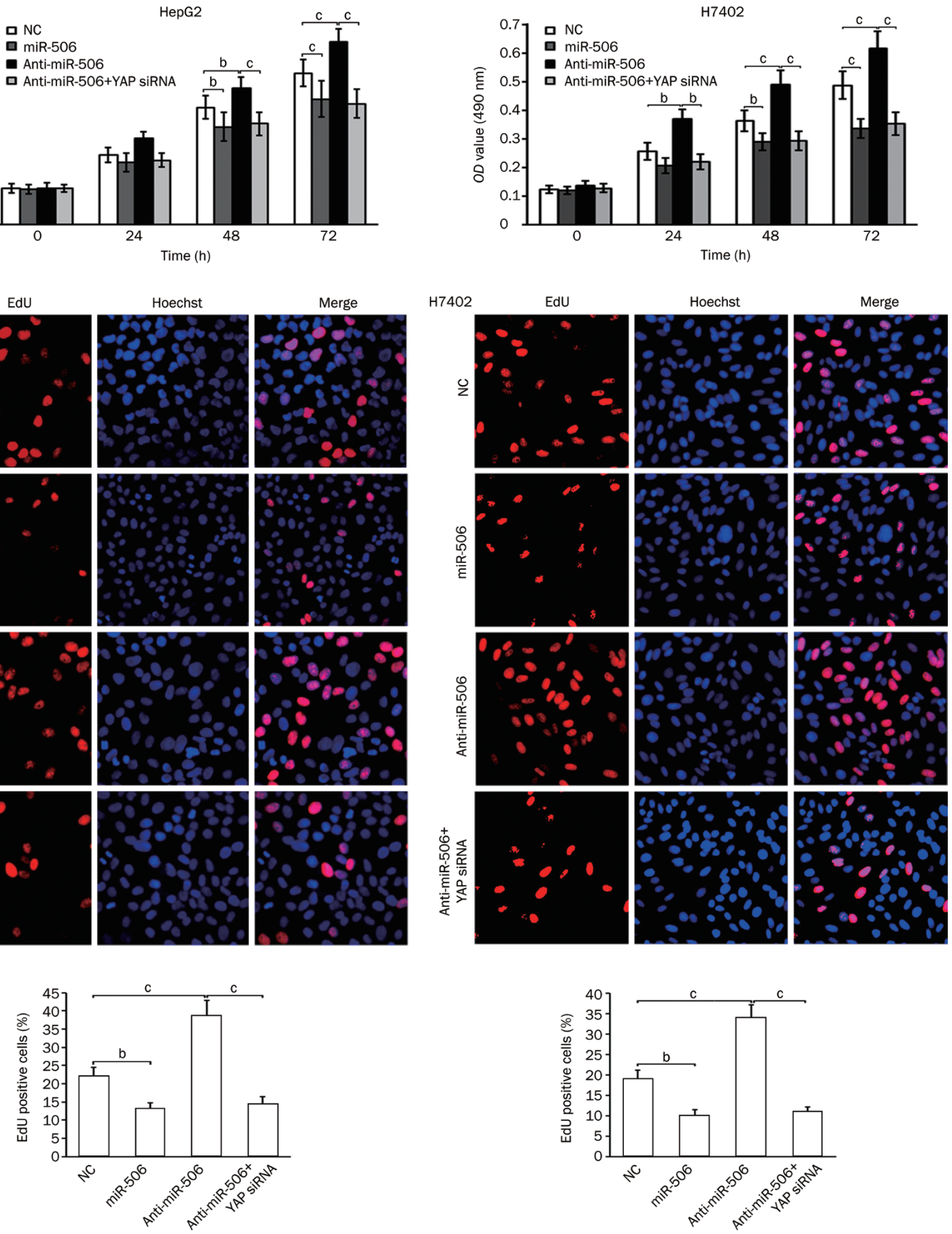

Figure 4. MiR-506 suppresses the proliferation of hepatoma cells by inhibiting YAP. (A and B) HepG2 and H7402 cells were transfected with NC (negative control), miR-506, anti-miR-506, or YAP siRNA. The effect of miR-506 on cell proliferation was determined by MTT and EdU assays. Statistically significant differences are indicated: ${ }^{\mathrm{b}} P<0.05$ and ${ }^{\mathrm{C}} P<0.01$; Student's $t$ test. 
tion of drug response, is regulated by miR-506 ${ }^{[34]}$. In addition, miR-506 regulates expression of PPAR alpha in hydroxycamptothecin-resistant human colon cancer cells ${ }^{[35]}$. MiR-184 posttranscriptionally regulates SOX7 expression and promotes proliferation in human hepatocellular carcinoma cells ${ }^{[36]}$. Our group has reported that miR-205 modulates abnormal lipid metabolism of hepatoma cells via targeting acyl-CoA synthetase long-chain family member 1 and 4 (ACSL1 and ACSL4) $\mathrm{mRNA}^{[37,38]}$. Based on our findings in this study, we conclude that miR-506 may act as a tumor suppressor miRNA in liver cancer by directly targeting YAP mRNA. Thus, our finding provides new insights into the mechanism by which miR-506 modulates proliferation of hepatoma cells.

\section{Acknowledgements}

This work was supported by grants from the National Natural Science Foundation of China (№ 81372186 and 81272218) and Support Program of National Science and Technology of China (No 2012BAI23B08).

\section{Author contribution}

Li-hong YE, Xiao-dong ZHANG, and Yue WANG designed the research, analyzed the data and wrote the paper; Yue WANG, Ming CUI, Bao-di SUN, and Fa-bao LIU performed the research; Li-hong YE and Xiao-dong ZHANG contributed new reagents or analytic tools.

\section{Abbreviations}

miRNA, microRNA; YAP, Yes-associated protein; HCC, hepatocellular carcinoma; CTGF, connective tissue growth factor; 3'UTR, 3' untranslated region; MTT, methylthiazolyldiphenyltetrazolium bromide; EdU, ethynyldeoxyuridine; qRT-PCR, quantitative real-time polymerase chain reaction; siRNAs, small interfering RNAs.

\section{Supplementary information}

Supplementary information is available at Acta Pharmacologica Sinica's website.

\section{References}

1 Gregory RI, Chendrimada TP, Cooch N, Shiekhattar R. Human RISC couples microRNA biogenesis and posttranscriptional gene silencing. Cell 2005; 123: 631-40.

2 Sassen S, Miska EA, Caldas C. MicroRNA: implications for cancer. Virchows Arch 2008; 452: 1-10.

3 Gregory PA, Bert AG, Paterson EL, Barry SC, Tsykin A, Farshid G, et al. The miR-200 family and miR-205 regulate epithelial to mesenchymal transition by targeting ZEB1 and SIP1. Nat Cell Biol 2008; 10: 593601.

4 Callegari E, Elamin BK, Sabbioni S, Gramantieri L, Negrini M. Role of microRNAs in hepatocellular carcinoma: a clinical perspective. Onco Targets Ther 2013; 6: 1167-78.

5 Lu R, Ji Z, Li X, Zhai Q, Zhao C, Jiang Z, et al. miR-145 functions as tumor suppressor and targets two oncogenes, ANGPT2 and NEDD9, in renal cell carcinoma. J Cancer Res Clin Oncol 2014; 140: 387-97.

6 Zheng SG, Xu HX, Liu LN. Management of hepatocellular carcinoma: The role of contrast-enhanced ultrasound. World J Radiol 2014; 6:
$7-14$.

7 Farazi PA, DePinho RA. Hepatocellular carcinoma pathogenesis: from genes to environment. Nat Rev Cancer 2006; 6: 674-87.

8 Zhang S, Shan C, Kong G, Du Y, Ye L, Zhang X. MicroRNA-520e suppresses growth of hepatoma cells by targeting the NF-kappaBinducing kinase (NIK). Oncogene 2012; 31: 3607-20.

9 Zhang T, Zhang J, Cui M, Liu F, You X, Du Y, et al. Hepatitis B virus $X$ protein inhibits tumor suppressor miR-205 through inducing hypermethylation of miR-205 promoter to enhance carcinogenesis. Neoplasia 2013; 15: 1282-91.

10 Yang D, Sun Y, Hu L, Zheng H, Ji P, Pecot CV, et al. Integrated analyses identify a master microRNA regulatory network for the mesenchymal subtype in serous ovarian cancer. Cancer Cell 2013; 23: 186-99.

11 Arora H, Qureshi R, Park WY. miR-506 regulates epithelial mesenchymal transition in breast cancer cell lines. PLoS One 2013; 8: e64273.

12 Yin M, Ren X, Zhang X, Luo Y, Wang G, Huang K, et al. Selective killing of lung cancer cells by miRNA-506 molecule through inhibiting NFkappaB p65 to evoke reactive oxygen species generation and p53 activation. Oncogene 2014. doi: 10.1038/onc.2013.597.

13 Xiao W, Wang J, Ou C, Zhang Y, Ma L, Weng W, et al. Mutual interaction between YAP and c-Myc is critical for carcinogenesis in liver cancer. Biochem Biophys Res Commun 2013; 439: 167-72.

14 Tschaharganeh DF, Chen X, Latzko P, Malz M, Gaida MM, Felix K, et al. Yes-associated protein up-regulates Jagged-1 and activates the Notch pathway in human hepatocellular carcinoma. Gastroenterology 2013; 144: 1530-42.

15 Bai N, Zhang C, Liang N, Zhang Z, Chang A, Yin J, et al. Yes-associated protein (YAP) increases chemosensitivity of hepatocellular carcinoma cells by modulation of p53. Cancer Biol Ther 2013; 14: 511-20.

16 Camargo FD, Gokhale S, Johnnidis JB, Fu D, Bell GW, Jaenisch R, et al. YAP1 increases organ size and expands undifferentiated progenitor cells. Curr Biol 2007; 17: 2054-60.

17 Lamar JM, Stern P, Liu H, Schindler JW, Jiang ZG, Hynes RO. The Hippo pathway target, YAP, promotes metastasis through its TEADinteraction domain. Proc Natl Acad Sci U S A 2012; 109: E2441-50.

18 Yu FX, Guan KL. The Hippo pathway: regulators and regulations. Genes Dev 2013; 27: 355-71.

19 Dong J, Feldmann G, Huang J, Wu S, Zhang N, Comerford SA, et al. Elucidation of a universal size-control mechanism in Drosophila and mammals. Cell 2007; 130: 1120-33.

20 Zhang T, Zhang J, You X, Liu Q, Du Y, Gao Y, et al. Hepatitis B virus $X$ protein modulates oncogene Yes-associated protein by CREB to promote growth of hepatoma cells. Hepatology 2012; 56: 2051-9.

21 Shan C, Xu F, Zhang S, You J, You X, Qiu L, et al. Hepatitis B virus X protein promotes liver cell proliferation via a positive cascade loop involving arachidonic acid metabolism and p-ERK1/2. Cell Res 2010; 20: $563-75$.

22 Liu-Chittenden Y, Huang B, Shim JS, Chen Q, Lee SJ, Anders RA, et al. Genetic and pharmacological disruption of the TEAD-YAP complex suppresses the oncogenic activity of YAP. Genes Dev 2012; 26: 1300-5.

23 Wang J, Park JS, Wei Y, Rajurkar M, Cotton JL, Fan Q, et al. TRIB2 acts downstream of Wnt/TCF in liver cancer cells to regulate YAP and C/EBPalpha function. Mol Cell 2013; 51: 211-25.

24 You X, Liu F, Zhang T, Li Y, Ye L, Zhang X. Hepatitis B virus X protein upregulates oncogene Rab18 to result in the dysregulation of lipogenesis and proliferation of hepatoma cells. Carcinogenesis 2013; 34: 1644-52.

25 You X, Liu F, Zhang T, Lv N, Liu Q, Shan C, et al. Hepatitis B virus X protein upregulates Lin28A/Lin28B through Sp-1/c-Myc to enhance 
the proliferation of hepatoma cells. Oncogene 2014; 33: 449-60.

26 Xu B, Li SH, Zheng R, Gao SB, Ding LH, Yin ZY, et al. Menin promotes hepatocellular carcinogenesis and epigenetically up-regulates Yap1 transcription. Proc Natl Acad Sci U S A 2013; 110: 17480-5.

27 Lau AN, Curtis SJ, Fillmore CM, Rowbotham SP, Mohseni M, Wagner $\mathrm{DE}$, et al. Tumor-propagating cells and Yap/Taz activity contribute to lung tumor progression and metastasis. EMBO J 2014; 33: 468-81.

28 Urtasun R, Latasa MU, Demartis MI, Balzani S, Goni S, GarciaIrigoyen $\mathrm{O}$, et al. Connective tissue growth factor autocriny in human hepatocellular carcinoma: oncogenic role and regulation by epidermal growth factor receptor/yes-associated protein-mediated activation. Hepatology 2011; 54: 2149-58.

29 Xu MZ, Yao TJ, Lee NP, Ng IO, Chan YT, Zender L, et al. Yes-associated protein is an independent prognostic marker in hepatocellular carcinoma. Cancer 2009; 115: 4576-85.

30 Li L, Wang J, Zhang Y, Zhang Y, Ma L, Weng W, et al. MEK1 promotes YAP and their interaction is critical for tumorigenesis in liver cancer. FEBS Lett 2013; 587: 3921-7.

31 Song T, Zhang X, Zhang L, Dong J, Cai W, Gao J, et al. miR-708 promotes the development of bladder carcinoma via direct repression of Caspase-2. J Cancer Res Clin Oncol 2013; 139: 1189-98.

32 Cao Z, Yoon JH, Nam SW, Lee JY, Park WS. PDCD4 expression inversely correlated with miR-21 levels in gastric cancers. J Cancer
Res Clin Oncol 2012; 138: 611-9.

33 Wang C, Gao C, Zhuang JL, Ding C, Wang Y. A combined approach identifies three mRNAs that are down-regulated by microRNA-29b and promote invasion ability in the breast cancer cell line MCF-7. J Cancer Res Clin Oncol 2012; 138: 2127-36.

34 Markova SM, Kroetz DL. ABCC4 is regulated by microRNA-124a and microRNA-506. Biochem Pharmacol 2014; 87: 515-22.

35 Tong JL, Zhang CP, Nie F, Xu XT, Zhu MM, Xiao SD, et al. MicroRNA 506 regulates expression of PPAR alpha in hydroxycamptothecinresistant human colon cancer cells. FEBS Lett 2011; 585: 3560-8.

36 Wu GG, Li WH, He WG, Jiang N, Zhang GX, Chen W, et al. Mir-184 post-transcriptionally regulates SOX7 expression and promotes cell proliferation in human hepatocellular carcinoma. PLoS One 2014; 9: e88796.

37 Cui M, Wang Y, Sun B, Xiao Z, Ye L, Zhang X. MiR-205 modulates abnormal lipid metabolism of hepatoma cells via targeting acyl-CoA synthetase long-chain family member 1 (ACSL1) mRNA. Biochem Biophys Res Commun 2014; 444: 270-5.

38 Cui M, Xiao Z, Sun B, Wang Y, Zheng M, Ye L, et al. Involvement of cholesterol in hepatitis $B$ virus $X$ protein-induced abnormal lipid metabolism of hepatoma cells via up-regulating miR-205-targeted ACSL4. Biochem Biophys Res Commun 2014; 445: 651-5. 